\title{
Different forms of Euler's theorem forhomogeneous functions to solve partial differential equation
}

\author{
Amit Kumar*1, Neha Sharma $^{2}$,Sonia Bajaj ${ }^{3}$ \\ ${ }^{1}$ Department of Mathematics Chandigarh University Gharuan ,Mohali. \\ ${ }^{2}$ Department of Mathematics Chandigarh University Gharuan ,Mohali. \\ ${ }^{3}$ Department of Mathematic Chandigarh University Gharuan ,Mohali. \\ ${ }^{1}$ Amitmalhotra224@gmail.com, ${ }^{2} 66$ nidhi66@gmail.com, ${ }^{3}$ soniamam53@gmail.com
}

Article History: Received: 11 January 2021; Accepted: 27 February 2021; Published online: 5 April 2021

Abstract:In this paper we will discuss Euler's theorem for homogenous functions to solve different order partial differential equations. We will see that how we can predict the solution of partial differential Equation using different approaches of this theorem. In fact we also consider the case when more than two independent variables will be involved in the partial differential equation whenever dependent functions will be homogenous functions. We will throw a light on one method called Ajayous rules to predict the solution of homogenous partial differential equation.

Keywords: Homogenous functions, Partial Differential equations, Ajayous Rules, order of partial differential equation

\section{Introduction}

Leonard Euler was one of the greatest mathematicians. His contribution is unforgettable. He has given so many powerful results of mathematics and one of those results is Euler's Theorem for homogenous function. Engineering students used to study this theorem because Partial differential equation is one of the important fields of mathematics that every engineer should know. Euler's theorem for homogenous function gives easy way todeal with specific type of partial differential equation which further deals with homogenous function. Using this result we can avoid cumbersome calculation and can get the final result in few steps only. In this paper will discuss extension and generalization of this theorem.

\section{Literature review}

[1]By Definition any function $z$ is said to be homogenousfunction of $x, y$ with degree ' $n$ ' if $z(t x, t y)=$ $t^{n} z(x, y)$ where $t>0$ and if $z$ is such type of function then

Euler's theorem states that

This is a partial differential equation of first order

$$
x z_{x}+y z_{y}=n z
$$

For second order partial differential equation

$$
x^{2} z_{x x}+y^{2} z_{y y}+2 x y z_{x y}=n(n-1) z
$$

Second order result has been generated by using first order result of this theorem.

Similarly for third order results becomes

$$
x^{3} \frac{\partial^{3} z}{\partial x^{3}}+3\left[x^{2} y \frac{\partial^{3} z}{\partial^{2} x \partial y}+y^{2} x \frac{\partial^{3} z}{\partial^{2} y \partial x}\right]+y^{3} \frac{\partial^{3} z}{\partial y^{3}}=n(n-1)(n-2) z
$$

We can expand this result for further higher order partial differential equation by using the lower partial differential equation.

[2] Now if $z$ is homogenous function of $x$ and $y$ with degree $n$ where every partial derivatives of order upto ' $m$ ' exist and are continuous then result which we will get is as follow

$$
\begin{aligned}
& x^{m} \frac{\partial^{m} z}{\partial x^{m}}+m x^{m-1} y \frac{\partial^{m} z}{\partial x^{m-1} \partial y}+\frac{1}{2} m(m-1) x^{m-2} y^{2} \frac{\partial^{m} z}{\partial x^{m-2} \partial y^{2}}+\ldots \ldots+m_{C_{k}} x^{m-k} y^{k} \frac{\partial^{m} z}{\partial x^{m-k} \partial y^{k}}+ \\
& m x y^{m-1} \frac{\partial^{m_{z}}}{\partial x \partial y^{m-1}}+y^{m} \frac{\partial^{m_{z}}}{\partial y^{m}}=n(n-1)(n-2) \ldots \ldots \ldots \ldots \ldots(n-m+1) z, \quad \text { for } m \leq
\end{aligned}
$$

$n$

From above result we can easily conclude that if $z$ is a homogenous function of $x$ and $y$ with degree ' $n$ 'whose all partial derivatives exists for order uto ' $n$ ' are continuous then

$$
\begin{gathered}
x^{n} \frac{\partial^{n} z}{\partial x^{n}}+n x^{n-1} y \\
\frac{\partial^{n} z}{\partial x^{n-1} \partial y}+n_{C_{2}} x^{n-2} y^{2} \frac{\partial^{n} z}{\partial x^{n-2} \partial y^{2}}+\ldots \ldots \ldots \ldots \ldots+n_{C_{k}} x^{n-k} y^{k} \frac{\partial^{n} z}{\partial x^{n-k} \partial y^{k}} \\
+\ldots \ldots \ldots \ldots \ldots \ldots \ldots \ldots \ldots y^{n-1} \frac{\partial^{n} z}{\partial x \partial y^{n-1}}+y^{n} \frac{\partial^{n} z}{\partial y^{n}}=n ! z \text { for } n \in N
\end{gathered}
$$

Next case would be when given function is composite one i.e. if $z=f(u)$ is homogenous function with 
Research Article

degree $n$ in $x$ and $y$ then Euler's theorem for homogenous function for first order will give this following result

$$
x \frac{\partial u}{\partial x}+y \frac{\partial u}{\partial y}=n \frac{f(u)}{f^{\prime}(u)}
$$

For second order if secondorder partial derivative exists and continuous then result is

$$
x^{2} \frac{\partial^{2} z}{\partial x^{2}}+2 x y \frac{\partial^{2} z}{\partial x \partial y}+y^{2} \frac{\partial^{2} z}{\partial y^{2}}=g(u) H_{1}(u), \text { where } g(u)=n \frac{f(u)}{f^{\prime}(u)} \text { and } H_{1}(u)=g^{\prime}(u)-1
$$

Similarly for third order if partial derivative upto third order exists and continuous then result is

$$
\begin{gathered}
x^{3} \frac{\partial^{3} u}{\partial x^{3}}+3\left[x^{2} y \frac{\partial^{3} u}{\partial x^{2} \partial y}+y^{2} x \frac{\partial^{3} u}{\partial y^{2} \partial x}\right]+y^{3} \frac{\partial^{3} u}{\partial y^{3}}=g(u)\left[g(u) H_{1}^{\prime}(u)+\left(g^{\prime}(u)-1\right)\left(g^{\prime}(u)-2\right)\right] \\
=\underset{g(u) H_{2}(u)}{\text { Where } H_{2}(u)=g(u) H_{1}^{\prime}(u)+\left(g^{\prime}(u)-1\right)\left(g^{\prime}(u)-2\right)}
\end{gathered}
$$

So in general if partial derivatives of u exists and continuous upto ' $m$ ' order where $z=f(u)$ is homogenous function with degree $n$ in $x$ and $y$ then

$$
\begin{gathered}
x^{m} \frac{\partial^{m} u}{\partial x^{m}}+m x^{m-1} y \frac{\partial^{m} u}{\partial x^{m-1} \partial y}+\frac{1}{2} m(m-1) x^{m-2} y^{2} \frac{\partial^{m} u}{\partial x^{m-2} \partial y^{2}}+\ldots \ldots \ldots \ldots \ldots+m_{C_{k}} x^{m-k} y^{k} \frac{\partial^{m} u}{\partial x^{m-k} \partial y^{k}} \\
\quad+\ldots \ldots \ldots \ldots \ldots \ldots \ldots m y^{m-1} \frac{\partial^{m} u}{\partial x \partial y^{m-1}}+y^{m} \frac{\partial^{m} u}{\partial y^{m}}=g(u) H_{m-1}(u) \text { for } m \leq n \\
\text { where } H_{m}=g(u) H_{m-1}^{\prime}(u)+\left(g^{\prime}(u)-1\right)\left(g^{\prime}(u)-2\right) \ldots \ldots \ldots \ldots\left(g^{\prime}(u)-m\right), m>1
\end{gathered}
$$

$$
g(u)=n \frac{f(u)}{f^{\prime}(u)} \text { and } H_{1}(u)=\left(g^{\prime}(u)-1\right)
$$

[3] Now next approach is Ajayous rules

Here one symbol is used i.e. $u_{x} p_{y} q u$ is differentiated with $x$ and $y$ partially ' $p$ ' times and ' $q$ ' times respectively.

Now Euler's theorem for homogenous function for higher order is as follows:

For second order it is

For third order it is

$$
x^{2} \frac{\partial^{2} u}{\partial x^{2}}+2 x y \frac{\partial^{2} u}{\partial x \partial y}+y^{2} \frac{\partial^{2} u}{\partial y^{2}}=n(n-1) u
$$

$$
x^{3} \frac{\partial^{3} u}{\partial x^{3}}+y^{3} \frac{\partial^{3} u}{\partial y^{3}}+2 x^{2} \frac{\partial^{2} u}{\partial x^{2}}+2 y^{2} \frac{\partial^{2} u}{\partial y^{2}}++3 x^{2} y \frac{\partial^{3} u}{\partial x^{2} \partial y}+3 x y^{2} \frac{\partial^{3} u}{\partial x \partial y^{2}}+4 x y \frac{\partial^{2} u}{\partial x \partial y}=n^{2}(n-1) u
$$

For fourth order

$$
\begin{aligned}
x^{4} \frac{\partial^{4} u}{\partial x^{4}}+y^{4} \frac{\partial^{4} u}{\partial y^{4}} & +5 x^{3} \frac{\partial^{3} u}{\partial x^{3}}+5 y^{3} \frac{\partial^{3} u}{\partial y^{3}}+4 x^{2} \frac{\partial^{2} u}{\partial x^{2}}+4 y^{2} \frac{\partial^{2} u}{\partial y^{2}}+15 x^{2} y \frac{\partial^{3} u}{\partial x^{2} \partial y}+15 x y^{2} \frac{\partial^{3} u}{\partial x \partial y^{2}}+4 x^{3} y \frac{\partial^{4} u}{\partial x^{3} \partial y} \\
& +4 x y^{3} \frac{\partial^{4} u}{\partial x \partial y^{3}}+6 x^{2} y^{2} \frac{\partial^{4} u}{\partial x^{2} \partial y^{2}}+8 x y \frac{\partial^{2} u}{\partial x \partial y}=n^{3}(n-1) u
\end{aligned}
$$

For fifth order

$$
\begin{aligned}
x^{5} \frac{\partial^{5} u}{\partial x^{5}}+y^{5} \frac{\partial^{5} u}{\partial y^{5}} & +9 x^{4} \frac{\partial^{4} u}{\partial x^{4}}+9 y^{4} \frac{\partial^{4} u}{\partial y^{4}}+19 x^{3} \frac{\partial^{3} u}{\partial x^{3}}+19 y^{3} \frac{\partial^{3} u}{\partial y^{3}}+8 x^{2} \frac{\partial^{2} u}{\partial x^{2}}+8 y^{2} \frac{\partial^{2} u}{\partial y^{2}}+10 x^{2} y^{3} \frac{\partial^{5} u}{\partial x^{2} \partial y^{3}} \\
& +10 x^{3} y^{2} \frac{\partial^{5} u}{\partial x^{3} \partial y^{2}}+36 x^{3} y \frac{\partial^{4} u}{\partial x^{3} \partial y}+36 x y^{3} \frac{\partial^{4} u}{\partial x \partial y^{3}}+57 x y^{2} \frac{\partial^{3} u}{\partial x \partial y^{2}}+57 x^{2} y \frac{\partial^{3} u}{\partial x^{2} \partial y} \\
& +5 x y^{4} \frac{\partial^{5} u}{\partial x \partial y^{4}}+5 x^{4} y \frac{\partial^{5} u}{\partial x^{4} \partial y}+54 x^{2} y^{2} \frac{\partial^{4} u}{\partial x^{2} \partial y^{2}}+16 x y \frac{\partial^{2} u}{\partial x \partial y}=n^{4}(n-1) u
\end{aligned}
$$

For sixth order

$$
\begin{aligned}
x^{6} \frac{\partial^{6} u}{\partial x^{6}}+y^{6} \frac{\partial^{6} u}{\partial y^{6}} & +14 x^{5} \frac{\partial^{5} u}{\partial x^{5}}+14 y^{5} \frac{\partial^{5} u}{\partial y^{5}}+55 x^{4} \frac{\partial^{4} u}{\partial x^{4}}+55 y^{4} \frac{\partial^{4} u}{\partial y^{4}}+65 x^{3} \frac{\partial^{3} u}{\partial x^{3}}+65 y^{3} \frac{\partial^{3} u}{\partial y^{3}}+16 x^{2} \frac{\partial^{2} u}{\partial x^{2}} \\
& +16 y^{2} \frac{\partial^{2} u}{\partial y^{2}}+20 x^{3} y^{3} \frac{\partial^{6} u}{\partial x^{3} \partial y^{3}}+140 x^{2} y^{3} \frac{\partial^{5} u}{\partial x^{2} \partial y^{3}}+140 x^{3} y^{2} \frac{\partial^{5} u}{\partial x^{3} \partial y^{2}}+239 x^{3} y \frac{\partial^{4} u}{\partial x^{3} \partial y} \\
& +239 x y^{3} \frac{\partial^{4} u}{\partial x \partial y^{3}}+15 x^{2} y^{4} \frac{\partial^{6} u}{\partial x^{2} \partial y^{4}}+15 x^{4} y^{2} \frac{\partial^{6} u}{\partial x^{4} \partial y^{2}}+70 x y^{4} \frac{\partial^{5} u}{\partial x \partial y^{4}}+70 x^{4} y \frac{\partial^{5} u}{\partial x^{4} \partial y} \\
& +6 x y^{5} \frac{\partial^{6} u}{\partial x \partial y^{5}}+6 x^{5} y \frac{\partial^{6} u}{\partial x^{5} \partial y}+195 x y^{2} \frac{\partial^{3} u}{\partial x \partial y^{2}}+195 x^{2} y \frac{\partial^{3} u}{\partial x^{2} \partial y}+330 x^{2} y^{2} \frac{\partial^{4} u}{\partial x^{2} \partial y^{2}} \\
& +32 x y \frac{\partial^{2} u}{\partial x \partial y}=n^{5}(n-1) u
\end{aligned}
$$


Now question is how to generalize this. For that we will use Ajayous rules. These rules are applicable only for order $N \geq 2$ and total number of rules are 5

Rule 1:

The Nth order partial equation of Euler's theorem is the form

Such that, $2 \leq p+q \leq N, 0 \leq p \leq N, 0 \leq q \leq N$

$$
\sum a_{j} x^{p} y^{q} u_{x} y^{q}=n^{N-1}(n-1) u
$$

Also $N \geq 2$, where $p, q \in W$

$$
j \text { varies from } 1 \text { to the total number of terms in the equation. }
$$

Now here

\begin{tabular}{|c|c|c|}
\hline $\begin{array}{c}\text { Rule } \\
\text { number }\end{array}$ & $\begin{array}{c}\text { Corresponding to the } \\
\text { terms }\end{array}$ & Value of aj constant coefficients \\
\hline 2 & $x^{2} u_{x^{2}} \& y^{2} u_{y^{2}}$ & $2^{N-2}$ \\
\hline 3 & $x y u_{x y}$ & $2^{N-1}$ \\
\hline 4 & $x^{3} u_{x^{3}} \& y^{3} u_{y^{3}}$ & $1-\frac{31}{4}(N-3)+\frac{181}{12}(N-3)^{2}$ \\
& & $-\frac{47}{6}(N-3)^{3}$ \\
& & $+\frac{23}{12}(N-3)^{4}$ \\
\hline
\end{tabular}

\section{Rule no. 5}

For other remaining terms in order to find the value of $a_{j}$ we need to compare expansion of $(x+y)^{N}$ with the left hand side of partial differential equations and then equate those terms which are similar.

We can verify these rules and compare with the above equation which has already been given for $N=1$ to 6

For ' $m$ ' Independent variables:

Now if $\mathrm{u}$ is a homogenous function of $x_{1}, x_{2}, x_{3}, \ldots \ldots \ldots \ldots \ldots, x_{m}$ variables with degree $n$ then Euler's theorem gives first order partial differential equation as follows

$$
x_{1} \frac{\partial u}{\partial x_{1}}+x_{2} \frac{\partial u}{\partial x_{2}}+x_{3} \frac{\partial u}{\partial x_{3}}+\ldots \ldots \ldots \ldots \ldots \ldots \ldots+x_{m} \frac{\partial u}{\partial x_{m}}=n u
$$

For higher order $\mathbf{N}=2$ results are given as

$$
\begin{aligned}
x_{1}^{2} \frac{\partial^{2} u}{\partial x_{1}^{2}}+x_{2}^{2} \frac{\partial^{2} u}{\partial x_{2}^{2}} & +x_{3}^{2} \frac{\partial^{2} u}{\partial x_{3}^{2}}+\ldots \ldots \ldots+x_{m}^{2} \frac{\partial^{2} u}{\partial x_{m}^{2}}+2 x_{1} x_{2} \frac{\partial^{2} u}{\partial x_{1} \partial x_{2}}+2 x_{1} x_{3} \frac{\partial^{2} u}{\partial x_{1} \partial x_{3}}+2 x_{1} x_{4} \frac{\partial^{2} u}{\partial x_{1} \partial x_{4}} \\
& +\ldots \ldots \ldots \ldots \ldots+2 x_{1} x_{m} \frac{\partial^{2} u}{\partial x_{1} \partial x_{m}}+2 x_{2} x_{3} \frac{\partial^{2} u}{\partial x_{2} \partial x_{3}}+2 x_{2} x_{4} \frac{\partial^{2} u}{\partial x_{2} \partial x_{4}}+2 x_{2} x_{5} \frac{\partial^{2} u}{\partial x_{2} \partial x_{5}} \\
& +\ldots \ldots \ldots \ldots \ldots+2 x_{2} x_{m} \frac{\partial^{2} u}{\partial x_{2} \partial x_{m}}+2 x_{3} x_{4} \frac{\partial^{2} u}{\partial x_{3} \partial x_{4}}+2 x_{3} x_{5} \frac{\partial^{2} u}{\partial x_{3} \partial x_{5}}+2 x_{3} x_{6} \frac{\partial^{2} u}{\partial x_{3} \partial x_{6}} \\
& +\ldots \ldots \ldots \ldots \ldots+2 x_{3} x_{m} \frac{\partial^{2} u}{\partial x_{3} \partial x_{m}}+\ldots \ldots \ldots \ldots \ldots \ldots+2 x_{m-1} x_{m} \frac{\partial^{2} u}{\partial x_{m-1} \partial x_{m}}=n(n-1) u
\end{aligned}
$$

$\sum_{i=1}^{\text {Or }} x_{i}^{2} \frac{\partial^{2} u}{\partial x_{i}^{2}}+2 x_{1} \sum_{i=2}^{m} x_{i} \frac{\partial^{2} u}{\partial x_{1} \partial x_{i}}+2 x_{2} \sum_{i=3}^{m} x_{i} \frac{\partial^{2} u}{\partial x_{2} \partial x_{i}}+\ldots \ldots \ldots \ldots . . . . .+2 x_{m-1} x_{m} \frac{\partial^{2} u}{\partial x_{m-1} \partial x_{m}}=n(n-1) u$

For $\mathbf{N}=\mathbf{2} \mathbf{m}=\mathbf{2}$ it becomes

For $\mathbf{N}=\mathbf{2 ,} \mathbf{m}=\mathbf{3}$

$$
\begin{gathered}
\sum_{i=1}^{2} x_{i}^{2} \frac{\partial^{2} u}{\partial x_{i}^{2}}+2 x_{1} \sum_{i=2}^{2} x_{i} \frac{\partial^{2} u}{\partial x_{1} \partial x_{i}}=n(n-1) u \\
x_{1}^{2} \frac{\partial^{2} u}{\partial x_{1}^{2}}+x_{2}^{2} \frac{\partial^{2} u}{\partial x_{2}^{2}}+2 x_{1} x_{2} \frac{\partial^{2} u}{\partial x_{1} \partial x_{2}}=n(n-1) u
\end{gathered}
$$




$$
\begin{gathered}
\sum_{i=1}^{3} x_{i}^{2} \frac{\partial^{2} u}{\partial x_{i}^{2}}+2 x_{1} \sum_{i=2}^{3} x_{i} \frac{\partial^{2} u}{\partial x_{1} \partial x_{i}}+2 x_{2} \sum_{i=3}^{3} x_{i} \frac{\partial^{2} u}{\partial x_{2} \partial x_{i}}=n(n-1) u \\
\text { i.e. } x_{1}^{2} \frac{\partial^{2} u}{\partial x_{1}^{2}}+x_{2}^{2} \frac{\partial^{2} u}{\partial x_{2}^{2}}+x_{3}^{2} \frac{\partial^{2} u}{\partial x_{3}^{2}}+2 x_{1} x_{2} \frac{\partial^{2} u}{\partial x_{1} \partial x_{2}}+2 x_{1} x_{3} \frac{\partial^{2} u}{\partial x_{1} \partial x_{3}}+2 x_{2} x_{3} \frac{\partial^{2} u}{\partial x_{2} \partial x_{3}}=n(n-1) u
\end{gathered}
$$

\section{For $\mathrm{N}=\mathbf{2 , \mathrm { m } = 4}$}

$$
\sum_{i=1}^{4} x_{i}^{2} \frac{\partial^{2} u}{\partial x_{i}^{2}}+2 x_{1} \sum_{i=2}^{4} x_{i} \frac{\partial^{2} u}{\partial x_{1} \partial x_{i}}+2 x_{2} \sum_{i=3}^{4} x_{i} \frac{\partial^{2} u}{\partial x_{2} \partial x_{i}}+2 x_{3} \sum_{i=4}^{4} x_{i} \frac{\partial^{2} u}{\partial x_{3} \partial x_{i}}=n(n-1) u
$$

$x_{1}^{2} \frac{\partial^{2} u}{\partial x_{1}^{2}}+x_{2}^{2} \frac{\partial^{2} u}{\partial x_{2}^{2}}+x_{3}^{2} \frac{\partial^{2} u}{\partial x_{3}^{2}}+x_{4}^{2} \frac{\partial^{2} u}{\partial x_{4}^{2}}+2 x_{1} x_{2} \frac{\partial^{2} u}{\partial x_{1} \partial x_{2}}+2 x_{1} x_{3} \frac{\partial^{2} u}{\partial x_{1} \partial x_{3}}+2 x_{2} x_{3} \frac{\partial^{2} u}{\partial x_{2} \partial x_{3}}+2 x_{2} x_{4} \frac{\partial^{2} u}{\partial x_{2} \partial x_{4}}$

$$
+2 x_{3} x_{4} \frac{\partial^{2} u}{\partial x_{3} \partial x_{4}}=n(n-1) u
$$

For $\mathbf{N}=3$ with $\mathbf{m}$ variables

$$
\begin{aligned}
\sum_{i=1}^{m} x_{i}^{3} \frac{\partial^{3} u}{\partial x_{i}^{3}}+2 \sum_{i=1}^{m} & x_{i}^{2} \frac{\partial^{2} u}{\partial x_{i}^{2}}+3 x_{1}^{2} \sum_{i=2}^{m} x_{i} \frac{\partial^{3} u}{\partial x_{1}^{2} \partial x_{i}}+3 x_{2}^{2} \sum_{i=1}^{m} x_{i} \frac{\partial^{3} u}{\partial x_{2}^{2} \partial x_{i}}+3 x_{3}^{2} \sum_{i=2}^{m} x_{i} \frac{\partial^{3} u}{\partial x_{3}^{2} \partial x_{i}}+\ldots \ldots \ldots \ldots \\
& +3 x_{m}^{2} x_{m-1} \frac{\partial^{3} u}{\partial x_{m}^{2} \partial x_{m-1}}+4 x_{1} \sum_{i=2}^{m} x_{i} \frac{\partial^{2} u}{\partial x_{1} \partial x_{i}}+4 x_{2} \sum_{i=3}^{m} x_{i} \frac{\partial^{2} u}{\partial x_{2} \partial x_{i}}+4 x_{3} \sum_{i=4}^{m} x_{i} \frac{\partial^{2} u}{\partial x_{3} \partial x_{i}} \\
& +\ldots \ldots \ldots \ldots \ldots+4 x_{m} x_{m-1} \frac{\partial^{3} u}{\partial x_{m} \partial x_{m-1}}+6 x_{1} x_{2} \sum_{i=3}^{m} x_{i} \frac{\partial^{2} u}{\partial x_{1} \partial x_{2} \partial x_{i}}+6 x_{1} x_{3} \sum_{i=4} x_{i} \frac{\partial^{2} u}{\partial x_{1} \partial x_{3} \partial x_{i}} \\
& +6 x_{1} x_{4} \sum_{i=5}^{m} x_{i} \frac{\partial^{2} u}{\partial x_{1} \partial x_{4} \partial x_{i}}+\ldots \ldots \ldots+6 x_{1} x_{m} x_{m-1} \frac{\partial^{3} u}{\partial x_{1} \partial x_{m} \partial x_{m-1}} \\
& +6 x_{2} x_{3} \sum_{i=3}^{m} x_{i} \frac{\partial^{2} u}{\partial x_{2} \partial x_{3} \partial x_{i}}+6 x_{2} x_{4} \sum_{i=4}^{m} x_{i} \frac{\partial^{2} u}{\partial x_{2} \partial x_{4} \partial x_{i}}+6 x_{2} x_{5} \sum_{i=5}^{m} x_{i} \frac{\partial^{2} u}{\partial x_{2} \partial x_{5} \partial x_{i}}+\ldots \ldots \ldots \ldots \\
& +6 x_{2} x_{m} x_{m-1} \frac{\partial^{3} u}{\partial x_{2} \partial x_{m} \partial x_{m-1}}+\ldots \ldots \ldots \ldots+6 x_{m} x_{m-1} x_{m-2} \frac{\partial^{3} u}{\partial x_{m} \partial x_{m-1} \partial x_{m-2}} \\
& =n^{2}(n-1) u
\end{aligned}
$$

Here summation is taken in such a manner that no term is of the form $x_{p}^{2} x_{q} u_{x_{q} x_{p}}{ }^{2}$ such that $p=q$ We can verify this result easily

\section{Conclusion}

Euler's theorem for homogenous function is a very important result which makes the way easier to solve the partial differential equations which deal with specific type of functions called homogenous function. We have discussed two approaches here first one was direct and second one was Ajayous rules. But one limitation of Ajayous rule is thatit isapplicable on partial differential equation of order two or more. We have studied generalization of this result for higher order and with $\mathrm{m}$ independent variables. This is a very useful tool to solve so many engineering problem involbing such type of partial differential equation.

\section{References}

1. Ramana B. V., Higher Engineering Mathmatics, Tata McGraw Hill, 2007.

2. A.P. Hiwarekar, Bulletin of the Marathwada Mathematical Society, Vol. 10, No. 2, December 2009, Pages $16\{19$.

3. Ajay Srinivas, International Journal of Scientific \& Engineering Research Volume 11, Issue 1, January-2020 ISSN 2229-5518

4. A Textbook of B.Sc. MATHEMATICS for 1ST Semester, BANGALORE UNIVERSITY BY G.K. RANGANATH.

5. Mathematics Prescribed textbook, 1ST PUC by GOVT. OF KARNATAKA.

6. Mathematics Prescribed textbook, 10TH STANDARD, NCERT, BY CENTRAL BOARD.

7. https://mathstackexchange.com

8. https://en.m.wikipedia.org/wiki. 
9. Wartikar P. N., Wartikar J. N., Text Book of Engineering Mathematics-

10. 1,PVG Pub, 2005.

11. Wartikar P. N., Wartikar J. N., Applied Mathematics Vol.I,PVG Pub, 2000. 